\title{
Constant Sign Solutions for Variable Exponent System Neumann Boundary Value Problems with Singular Coefficient
}

\begin{abstract}
Xianbin Wu
Junior College, Zhejiang Wanli University, Ningbo, Zhejiang 315100, China

Correspondence should be addressed to Xianbin Wu; wxb3210@zwu.edu.cn

Received 14 November 2013; Accepted 22 February 2014; Published 7 April 2014

Academic Editor: Leszek Gasinski

Copyright (c) 2014 Xianbin Wu. This is an open access article distributed under the Creative Commons Attribution License, which permits unrestricted use, distribution, and reproduction in any medium, provided the original work is properly cited.

We deal with the existence of constant sign solutions for the following variable exponent system Neumann boundary value problem: $-\operatorname{div}\left(|\nabla u|^{p(x)-2} \nabla u\right)+\lambda|u|^{p(x)-2} u=F_{u}(x, u, v)$ in $\Omega,-\operatorname{div}\left(|\nabla v|^{q(x)-2} \nabla v\right)+\lambda|v|^{q(x)-2} v=F_{v}(x, u, v)$ in $\Omega, \partial u / \partial \gamma=0=\partial v / \partial \gamma$ on $\partial \Omega$. We give several sufficient conditions for the existence of the constant sign solutions, when $F(x, \cdot, \cdot)$ satisfies neither sub- $(p(x), q(x))$ growth condition, nor Ambrosetti-Rabinowitz condition (subcritical). In particular, we obtain the existence of eight constant sign solutions.
\end{abstract}

\section{Introduction}

In recent years, there is a lot of interest in the study of various mathematical problems with variable exponent (see [1-32]). We refer readers to $[1,23,31,33]$ for the background of these problems. On the existence of solutions for elliptic systems with variable exponent, we refer to $[15,25,27]$. In this paper, we consider the existence of constant sign solutions for the following problem:

$$
\begin{aligned}
& -\operatorname{div}\left(|\nabla u|^{p(x)-2} \nabla u\right)+\lambda|u|^{p(x)-2} u=F_{u}(x, u, v) \quad \text { in } \Omega \text {, } \\
& -\operatorname{div}\left(|\nabla v|^{q(x)-2} \nabla v\right)+\lambda|v|^{q(x)-2} v=F_{v}(x, u, v) \quad \text { in } \Omega, \\
& \frac{\partial u}{\partial \gamma}=0=\frac{\partial v}{\partial \gamma} \quad \text { on } \partial \Omega \text {, }
\end{aligned}
$$

where $\Omega \subset \mathbb{R}^{N}$ is an open bounded domain and $\partial \Omega$ possesses the cone property, $p, q \in C(\bar{\Omega})$ and $p(x), q(x)>1$, $-\triangle_{p(x)} u:=-\operatorname{div}\left(|\nabla u|^{p(x)-2} \nabla u\right)$ is called the $p(x)$-Laplacian, $\lambda \geq 1$ is a positive parameter, and $\gamma$ is the outward unit normal to $\partial \Omega$. F satisfies

$$
F(x, s, t)=a(x) G(x, s, t), \quad \forall(x, s, t) \in \bar{\Omega} \times \mathbb{R} \times \mathbb{R} .
$$

We make the following assumption.

$\left(\mathrm{A}_{0}\right) a \in L^{r(\cdot)}(\Omega)$ is a nontrivial nonnegative singular coefficient; $G \in C^{1}\left(\bar{\Omega} \times \mathbb{R}^{2} \rightarrow \mathbb{R}\right)$ and satisfies

$$
\begin{array}{r}
\left|G_{s}(x, s, t)\right| \leq C\left(|s|^{\alpha(x)-1}+|t|^{\beta(x) / \alpha^{0}(x)}+1\right), \\
\forall x \in \bar{\Omega}, \\
\left|G_{t}(x, s, t)\right| \leq C\left(|t|^{\beta(x)-1}+|s|^{\alpha(x) / \beta^{0}(x)}+1\right), \\
\forall x \in \bar{\Omega},
\end{array}
$$

where $r, \alpha, \beta \in C(\bar{\Omega}), r(x)>1,1<\alpha(x)<p^{*}(x), 1<\beta(x)<$ $q^{*}(x)$ on $\bar{\Omega}, G_{s}=(\partial / \partial s) G, G_{t}=(\partial / \partial t) G$, and satisfies

$$
r(x)>\max \left\{\left(\frac{p^{*}(x)}{\alpha(x)}\right)^{0},(p(x))^{0},\left(\frac{q^{*}(x)}{\beta(x)}\right)^{0},(q(x))^{0}\right\},
$$

where the notation $f^{0}(x)$ means the conjugate function of $f(x)$, namely, $(1 / f(x))+\left(1 / f^{0}(x)\right) \equiv 1$, and

$$
p^{*}(x)= \begin{cases}\frac{N p(x)}{(N-p(x))}, & p(x)<N, \\ \infty, & p(x) \geq N .\end{cases}
$$


When $p(\cdot) \equiv p$ (a constant), $p(x)$-Laplacian is the usual $p$-Laplacian. The $p(x)$-Laplacian is nonhomogeneity. Because of its nonhomogeneity, the $p(x)$-Laplacian possesses more complicated nonlinearity than the $p$-Laplacian. Many results and methods for $p$-Laplacian problems do not hold for $p(x)$-Laplacian problems anymore. For the following examples.

$\left(1^{0}\right)$ if $\Omega \subset \mathbb{R}^{N}$ is an open bounded domain, the Rayleigh quotient

$$
\lambda_{p(\cdot)}=\inf _{u \in W_{0}^{1, p(\cdot)}(\Omega) \backslash\{0\}} \frac{\int_{\Omega}(1 / p(x))|\nabla u|^{p(x)} d x}{\int_{\Omega}(1 / p(x))|u|^{p(x)} d x}
$$

is zero in general. Only under some special conditions, we have $\lambda_{p(\cdot)}>0$. For example, $\lambda_{p(\cdot)}>0$ if and only if $p(\cdot)$ is monotone in one-dimensional case (i.e., $N=1$ ) (see [11]). It is well known that the fact that $\lambda_{p}>0$ is very important in the study of $p$-Laplacian problems. For instance, in [34], the first eigenvalue and first eigenfunction are used to discuss the existence of positive solutions of $p$-Laplacian problems successfully. But the $p(x)$-Laplacian does not have the first eigenvalue and first eigenfunction in general.

$\left(2^{0}\right)$ The norm in $L^{p(\cdot)}(\Omega)$ is of Luxemburg type (we will explain later in Section 2). It is easy to see that $\int_{\Omega}|u|^{p(x)} d x=$ $|u|_{p(\cdot)}^{p(\xi)}$ for some $\xi \in \bar{\Omega}$. Hence the integral and the norm cannot keep the constant exponent relationship. It implies that we will have more difficulties in the study of $p(x)$ Laplacian problems. For example, it is very difficult to get the best Sobolev imbedding constant when we deal with the critical Sobolev exponent problems. Even if the best Sobolev imbedding constant could be obtained, it is also very hard to be applied to study the critical exponent problems.

$\left(3^{0}\right)$ In [35], the authors applied the homogeneous transformation method to discuss the existence of positive solutions for a class of superlinear semipositon systems. Nonetheless, the $p(x)$-Laplacian is nonhomogeneity; this method is very hard to be used on the $p(x)$-Laplacian problems.

On the existence of constant sign solutions of $p$-Laplacian problems, we refer to [35-40]. On the results of the constant sign solutions of variable exponent differential equations, we refer to $[14,19,29]$.

Regarding the existence of solutions of $(\mathrm{P})$, if $F(x, \cdot, \cdot)$ satisfies the sub- $\left(p^{-}, q^{-}\right)$growth condition, that is,

$$
\max _{x \in \bar{\Omega}} \alpha(x)<\min _{x \in \bar{\Omega}} p(x), \quad \max _{x \in \bar{\Omega}} \beta(x)<\min _{x \in \bar{\Omega}} q(x),
$$

then the corresponding functional of $(\mathrm{P})$ is coercive; if $F(x, \cdot, \cdot)$ satisfies the super- $\left(p^{+}, q^{+}\right)$growth condition (subcritical), that is, the following Ambrosetti-Rabinowitz condition:

$$
\begin{array}{r}
0<G(x, s, t) \leq \frac{s}{\theta_{1}} \frac{\partial}{\partial s} G(x, s, t)+\frac{t}{\theta_{2}} \frac{\partial}{\partial t} G(x, s, t), \\
\forall x \in \bar{\Omega},
\end{array}
$$

where positive constants $\theta_{1}$ and $\theta_{2}$ satisfy

$$
\max _{x \in \bar{\Omega}} p(x)<\theta_{1}<p^{*}(x), \quad \max _{x \in \bar{\Omega}} q(x)<\theta_{2}<q^{*}(x),
$$

then the corresponding functional of $(\mathrm{P})$ satisfies PalaisSmale conditions (see $[15,25])$. If $F(x, \cdot, \cdot)$ satisfies the subcritical growth condition, but it satisfies neither the sub- $\left(p^{-}, q^{-}\right)$ growth condition nor the super- $\left(p^{+}, q^{+}\right)$growth condition, then it would be difficult to testify that the corresponding functional is coercive or satisfying Palais-Smale conditions; the results in this case are rare.

In this paper, we deal with the existence of constant sign solutions of the problem $(\mathrm{P})$, when the corresponding functional neither is coercive nor satisfies Ambrosetti-Rabinowitz condition. For example, we discuss the existence of solutions of $(\mathrm{P})$, when $F$ satisfies sub- $(p(x), q(x))$ growth condition near the origin in local; that is, the following condition

$$
\begin{aligned}
& F(x, s, t) \geq \sigma\left(|s|^{\epsilon_{1}(x)}+|t|^{\epsilon_{2}(x)}\right), \\
& \forall(x, s, t) \in \bar{\Omega}_{0} \times(0, \delta) \times(0, \delta),
\end{aligned}
$$

where $1<\epsilon_{1}(x)<p(x), \quad 1<\epsilon_{2}(x)<q(x) \quad$ on $\bar{\Omega}_{0}$,

or $F$ satisfies super- $(p(x), q(x))$ growth condition in local (subcritical growth); that is, the following condition:

$$
\begin{array}{r}
0<G(x, s, t) \leq \\
\frac{s}{\theta_{1}(x)} \frac{\partial}{\partial s} G(x, s, t)+\frac{t}{\theta_{2}(x)} \frac{\partial}{\partial t} G(x, s, t), \\
x \in \bar{\Omega}, \quad s>0, \quad t>0, \quad|s|+|t| \geq M,
\end{array}
$$

where positive functions $\theta_{1}(\cdot)$ and $\theta_{2}(\cdot)$ satisfy

$$
\begin{array}{r}
p(x)<\theta_{1}(x)<p^{*}(x), \quad q(x)<\theta_{2}(x)<q^{*}(x), \\
\forall x \in \bar{\Omega} .
\end{array}
$$

In particular, we get the existence of eight constant sign solutions of $(\mathrm{P})$.

This paper is divided into four sections. In Section 2, we introduce some basic properties of the variable exponent Lebesgue-Sobolev spaces. In Section 3, several properties of $p(x)$-Laplacian are presented. In Section 4, we give the existence results of constant sign solutions of problem (P).

\section{Preliminary Results and Notations}

Throughout this paper, the letters $c, c_{i}, C_{i}, i=1,2, \ldots$, denote positive constants which may vary from line to line but are independent of the terms which will take part in any limit process.

In order to discuss the problem $(\mathrm{P})$, we need some theories on space $W^{1, p(\cdot)}(\Omega)$ which we call variable exponent Sobolev space. Firstly, we state some basic properties of spaces 
$W^{1, p(\cdot)}(\Omega)$ and $p(x)$-Laplacian which we will use later (for details, see $[6,10,12,13])$. Write

$$
\begin{aligned}
C_{+}(\bar{\Omega}) & =\{h \mid h \in C(\bar{\Omega}), h(x) \geq 1 \text { for } x \in \bar{\Omega}\}, \\
h^{+} & =\underset{x \in \Omega}{\operatorname{ess} \sup _{x}} h(x), \quad h^{-}=\underset{x \in \Omega}{\operatorname{ess} \inf } h(x),
\end{aligned}
$$$$
\text { for any } h \in L^{\infty}(\Omega) \text {; }
$$

$S(\Omega)=\{u \mid u$ is a measurable real-valued function in $\Omega\}$,

$$
L^{p(\cdot)}(\Omega)=\left\{\left.u \in S(\Omega)\left|\int_{\Omega}\right| u(x)\right|^{p(x)} d x<\infty\right\} .
$$

We introduce the norm on $L^{p(\cdot)}(\Omega)$ by

$$
|u|_{p(\cdot)}=\inf \left\{\lambda>\left.0\left|\int_{\Omega}\right| \frac{u(x)}{\lambda}\right|^{p(x)} d x \leq 1\right\},
$$

and $\left(L^{p(\cdot)}(\Omega),|\cdot|_{p(\cdot)}\right)$ becomes a Banach space; we call it variable exponent Lebesgue space.

Proposition 1 (see [6]). (i) The space $\left(L^{p(\cdot)}(\Omega),|\cdot|_{p(\cdot)}\right)$ is a separable, uniform convex Banach space, and its conjugate space is $L^{p^{0}(\cdot)}(\Omega)$, where $(1 / p(x))+\left(1 / p^{0}(x)\right) \equiv 1$. For any $u \in L^{p(\cdot)}(\Omega)$ and $v \in L^{p^{0}(\cdot)}(\Omega)$, one has

$$
\left|\int_{\Omega} u v d x\right| \leq\left(\frac{1}{p^{-}}+\frac{1}{\left(p^{0}\right)^{-}}\right)|u|_{p(\cdot)}|v|_{p^{0}(\cdot)} .
$$

(ii) If $p_{1}, p_{2} \in C_{+}(\bar{\Omega}), p_{1}(x) \leq p_{2}(x)$ for any $x \in \bar{\Omega}$, then $L^{p_{2}(\cdot)}(\Omega) \subset L^{p_{1}(\cdot)}(\Omega)$, and the imbedding is continuous.

Denote $Y=\prod_{i=1}^{k} L^{p_{i}(\cdot)}(\Omega)$ with the norm

$$
\|y\|_{Y}=\sum_{i=1}^{k}\left|y^{i}\right|_{p_{i}(\cdot)}, \quad \forall y=\left(y^{1}, \ldots, y^{k}\right) \in Y,
$$

where $p_{i}(x) \in C_{+}(\bar{\Omega}), i=1, \ldots, m$; then $Y$ is a Banach space.

Proposition 2. If $f(x, y): \Omega \times \mathbb{R}^{k} \rightarrow \mathbb{R}^{m}$ is a Caratheodory function, that is, $f$ satisfies the following:

(i) for a.e. $x \in \Omega, y \rightarrow f(x, y)$ is a continuous function from $\mathbb{R}^{k}$ to $\mathbb{R}^{m}$;

(ii) for any $y \in \mathbb{R}^{k}, x \rightarrow f(x, y)$ is measurable.

If there exist $\beta(x), p_{1}(x), \ldots, p_{k}(x) \in C_{+}(\bar{\Omega}), \rho(x) \in$ $L^{\beta(\cdot)}(\Omega)$, and positive constant $c>0$ such that

$$
\begin{array}{r}
|f(x, y)| \leq \rho(x)+c \sum_{i=1}^{k}\left|y_{i}\right|^{p_{i}(x) / \beta(x)} \\
\text { for any } x \in \Omega, y \in \mathbb{R}^{k},
\end{array}
$$

then the Nemytsky operator from $Y$ to $\left(L^{\beta(\cdot)}(\Omega)\right)^{m}$ defined by $\left(N_{f} u\right)(x)=f(x, u(x))$ is a continuous and bounded operator.
Proof. Similar to the proof of [41], we omit it here.

Proposition 3 (see [6]). If one denotes

$$
\rho(u)=\int_{\Omega}|u|^{p(x)} d x, \quad \forall u \in L^{p(\cdot)}(\Omega),
$$

then

(i) $|u|_{p(\cdot)}<1(=1 ;>1) \Leftrightarrow \rho(u)<1(=1 ;>1)$;

(ii) $|u|_{p(\cdot)}>1 \Rightarrow|u|_{p^{(\cdot)}}^{p^{-}} \leq \rho(u) \leq|u|_{p(\cdot)}^{p^{+}} ;|u|_{p(\cdot)}<1 \Rightarrow$ $|u|_{p(\cdot)}^{p^{-}} \geq \rho(u) \geq|u|_{p(\cdot)}^{p^{+}}$

(iii) $|u|_{p(\cdot)} \rightarrow 0 \Leftrightarrow \rho(u) \rightarrow 0 ;|u|_{p(\cdot)} \rightarrow \infty \Leftrightarrow \rho(u) \rightarrow$ $\infty$.

Proposition 4 (see [6]). If $u, u_{n} \in L^{p(\cdot)}(\Omega), n=1,2, \ldots$, then the following statements are equivalent to each other:

(1) $\lim _{n \rightarrow \infty}\left|u_{n}-u\right|_{p(\cdot)}=0$;

(2) $\lim _{n \rightarrow \infty} \rho\left(u_{n}-u\right)=0$;

(3) $u_{n} \rightarrow u$ in measure in $\Omega$ and $\lim _{n \rightarrow \infty} \rho\left(u_{n}\right)=\rho(u)$.

The spaces $W^{1, p(\cdot)}(\Omega)$ and $W^{1, q(\cdot)}(\Omega)$ are defined by

$$
\begin{aligned}
& W^{1, p(\cdot)}(\Omega)=\left\{u \in L^{p(\cdot)}(\Omega)|| \nabla u \mid \in L^{p(\cdot)}(\Omega)\right\}, \\
& W^{1, q(\cdot)}(\Omega)=\left\{v \in L^{q(\cdot)}(\Omega)|| \nabla v \mid \in L^{q(\cdot)}(\Omega)\right\},
\end{aligned}
$$

and endowed with the following norm:

$$
\begin{aligned}
& \|u\|_{p(\cdot)}=\inf \left\{\mu>\left.0\left|\int_{\Omega}\right| \frac{\nabla u}{\mu}\right|^{p(x)} d x+\int_{\Omega}\left|\frac{u(x)}{\mu}\right|^{p(x)} d x \leq 1\right\}, \\
& \|u\|_{q(\cdot)}=\inf \left\{\mu>\left.0\left|\int_{\Omega}\right| \frac{\nabla u}{\mu}\right|^{q(x)} d x+\int_{\Omega}\left|\frac{u(x)}{\mu}\right|^{q(x)} d x \leq 1\right\} .
\end{aligned}
$$

We denote by $W_{0}^{1, p(\cdot)}(\Omega)$ the closure of $C_{0}^{\infty}(\Omega)$ in $W^{1, p(\cdot)}(\Omega)$.

Proposition 5 (see [6]). (i) $W^{1, p(\cdot)}(\Omega)$ is a separable reflexive Banach space.

(ii) If $\beta \in C_{+}(\bar{\Omega})$ and $\beta(x)<p^{*}(x)$ for any $x \in \bar{\Omega}$, then the imbedding from $W^{1, p(\cdot)}(\Omega)$ to $L^{\beta(\cdot)}(\Omega)$ is compact and continuous. Define

Let $\beta \in C_{+}(\bar{\Omega}), \mu \in S(\Omega)$, and $\mu(x)>0$ for a.e. $x \in \Omega$.

$$
L_{\mu(\cdot)}^{\beta(\cdot)}(\Omega)=\left\{\left.u\left|u \in S(\Omega), \int_{\Omega} \mu(x)\right| u(x)\right|^{\beta(x)} d x<\infty\right\},
$$

with the norm

$$
\begin{aligned}
|u|_{L_{\mu(\cdot)}^{\beta(\cdot)}(\Omega)} & =|u|_{(\beta(\cdot), \mu(\cdot))} \\
& =\inf \left\{\lambda>\left.0\left|\int_{\Omega} \mu(x)\right| \frac{u(x)}{\lambda}\right|^{\beta(x)} d x \leq 1\right\} ;
\end{aligned}
$$

then $L_{\mu(\cdot)}^{\beta(\cdot)}(\Omega)$ is a Banach space. 
Proposition 6 (see [12]). Assume that the boundary of $\Omega$ possesses the cone property and $1<p \in C(\bar{\Omega})$. Suppose that $\mu \in L^{r(\cdot)}(\Omega), \mu(x)>0$ for a.e. $x \in \Omega, r \in C(\bar{\Omega})$, and $r^{-}>1$. If $\beta \in C_{+}(\bar{\Omega})$ and

$$
1 \leq \beta(x)<\frac{r(x)-1}{r(x)} p^{*}(x), \quad \forall x \in \bar{\Omega} ;
$$

then the embedding $W^{1, p(\cdot)}(\Omega) \hookrightarrow L_{\mu(\cdot)}^{\beta(\cdot)}(\Omega)$ is compact.

Denote $X=W^{1, p(\cdot)}(\Omega) \times W^{1, q(\cdot)}(\Omega)$. The norm $\|\cdot\|$ on $X$ is defined by

$$
\|(u, v)\|=\max \left\{\|u\|_{p(\cdot)},\|v\|_{q(\cdot)}\right\} .
$$

For any $(u, v)$ and $(\varphi, \psi)$ in $X$, let

$$
\begin{aligned}
\Phi_{1}(u) & =\int_{\Omega} \frac{1}{p(x)}\left(|\nabla u|^{p(x)} d x+\lambda|u|^{p(x)}\right) d x, \\
\Phi_{2}(v) & =\int_{\Omega} \frac{1}{q(x)}\left(|\nabla v|^{q(x)} d x+\lambda|v|^{q(x)}\right) d x, \\
\Phi(u, v) & =\Phi_{1}(u)+\Phi_{2}(v), \\
\Psi(u, v) & =\int_{\Omega} F(x, u, v) d x .
\end{aligned}
$$

Then

$$
\begin{aligned}
& \Phi^{\prime}(u, v)(\varphi, \psi)=D_{1} \Phi(u, v)(\varphi)+D_{2} \Phi(u, v)(\psi), \\
& \Psi^{\prime}(u, v)(\varphi, \psi)=D_{1} \Psi(u, v)(\varphi)+D_{2} \Psi(u, v)(\psi),
\end{aligned}
$$

where

$$
\begin{aligned}
& D_{1} \Phi(u, v)(\varphi)=\int_{\Omega}|\nabla u|^{p(x)-2} \nabla u \nabla \varphi d x+\int_{\Omega} \lambda|u|^{p(x)-2} u \varphi d x \\
&=\Phi_{1}^{\prime}(u)(\varphi), \\
& D_{2} \Phi(u, v)(\psi)=\int_{\Omega}|\nabla v|^{q(x)-2} \nabla v \nabla \psi d x+\int_{\Omega} \lambda|v|^{q(x)-2} v \psi d x \\
&=\Phi_{2}^{\prime}(v)(\psi), \\
& D_{1} \Psi(u, v)(\varphi)=\int_{\Omega} \frac{\partial}{\partial u} F(x, u, v) \varphi d x \\
& D_{2} \Psi(u, v)(\psi)=\int_{\Omega} \frac{\partial}{\partial v} F(x, u, v) \psi d x .
\end{aligned}
$$

The integral functional associated with the problem $(\mathrm{P})$ is

$$
J(u, v)=\Phi(u, v)-\Psi(u, v) .
$$

Without loss of generality, we may assume that $G(x, 0,0)=0, \forall x \in \bar{\Omega}$. Obviously, We have

$$
G(x, u, v)=\int_{0}^{1}\left[u \partial_{2} G(x, t u, t v)+v \partial_{3} G(x, t u, t v)\right] d t,
$$$$
\forall x \in \bar{\Omega} \text {, }
$$

where $\partial_{j}$ denotes the partial derivative of $G$ with respect to its $j$ th variable; then the condition $\left(\mathrm{A}_{0}\right)$ holds

$$
|G(x, u, v)| \leq c\left(|u|^{\alpha(x)}+|v|^{\beta(x)}+1\right), \quad \forall x \in \bar{\Omega} .
$$

From Propositions 2 and 6 and condition $\left(\mathrm{A}_{0}\right)$, it is easy to see that $J \in C^{1}(X, \mathbb{R})$ and satisfies

$$
J^{\prime}(u, v)(\varphi, \psi)=D_{1} J(u, v)(\varphi)+D_{2} J(u, v)(\psi),
$$

where

$$
\begin{aligned}
& D_{1} J(u, v)(\varphi)=D_{1} \Phi(u, v)(\varphi)-D_{1} \Psi(u, v)(\varphi), \\
& D_{2} J(u, v)(\psi)=D_{2} \Phi(u, v)(\psi)-D_{2} \Psi(u, v)(\psi) .
\end{aligned}
$$

We say $(u, v) \in X$ is a critical point of $J$ if

$$
J^{\prime}(u, v)(\varphi, \psi)=0, \quad \forall(\varphi, \psi) \in X .
$$

The dual space of $X$ will be denoted by $X^{*}$; then for any $H \in X^{*}$, there exists $f \in\left(W^{1, p(\cdot)}(\Omega)\right)^{*}, g \in\left(W^{1, q(\cdot)}(\Omega)\right)^{*}$ such that $H(u, v)=f(u)+g(v)$. We denote $\|\cdot\|_{*},\|\cdot\|_{*, p(\cdot)}$, and $\|\cdot\|_{*, q(\cdot)}$ to be the norms of $X^{*},\left(W^{1, p(\cdot)}(\Omega)\right)^{*}$, and $\left(W^{1, q(\cdot)}(\Omega)\right)^{*}$, respectively. It is well known,

$$
\|H\|_{*}=\|f\|_{*, p(\cdot)}+\|g\|_{*, q(\cdot)},
$$

and $X^{*}=\left(W^{1, p(\cdot)}(\Omega)\right)^{*} \times\left(W^{1, q(\cdot)}(\Omega)\right)^{*}$. Therefore

$$
\left\|J^{\prime}(u, v)\right\|_{*}=\left\|D_{1} J(u, v)\right\|_{*, p(\cdot)}+\left\|D_{2} J(u, v)\right\|_{*, q(\cdot)} .
$$

Proposition 7. (i) If G satisfies

$$
\begin{array}{r}
G(x, s, t) \geq \frac{1}{\theta_{1}} s G_{s}(x, s, t)+\frac{1}{\theta_{2}} t G_{t}(x, s, t) \geq 0 \\
\text { for } x \in \bar{\Omega}, \quad|s|^{\theta_{1}}+|t|^{\theta_{2}} \geq 2 M,
\end{array}
$$

then $G(x, s, t) \leq c_{1}\left[\left(|s|^{\theta_{1}}+|t|^{\theta_{2}}\right)+1\right], \forall(x, s, t) \in \bar{\Omega} \times \mathbb{R} \times \mathbb{R}$.

(ii) If $G$ satisfies

$$
\begin{array}{r}
0<G(x, s, t) \leq \frac{1}{\theta_{1}} s G_{s}(x, s, t)+\frac{1}{\theta_{2}} t G_{t}(x, s, t) \\
\text { for } x \in \bar{\Omega}, \quad|s|^{\theta_{1}}+|t|^{\theta_{2}} \geq 2 M,
\end{array}
$$

then $G(x, s, v) \geq c_{2}\left[\left(|s|^{\theta_{1}}+|t|^{\theta_{2}}\right)-1\right], \forall(x, s, t) \in \bar{\Omega} \times \mathbb{R} \times \mathbb{R}$.

Proof. (i) Similar to the proof of [15], we omit it here.

\section{Properties of Operators}

In this section, we will discuss the properties of $p(x)$ Laplacian and Nemytsky operator.

From Propositions 2 and 6, we can easily see that $\Phi \in$ $C^{1}(X, \mathbb{R})$. 
Proposition 8 (see [25]). (i) $\Phi$ is a convex functional;

(ii) $\Phi^{\prime}$ is strictly monotone; that is, for any $\left(u_{1}, v_{1}\right)$, $\left(u_{2}, v_{2}\right) \in X$ with $\left(u_{1}, v_{1}\right) \neq\left(u_{2}, v_{2}\right)$, we have

$$
\left(\Phi^{\prime}\left(u_{1}, v_{1}\right)-\Phi^{\prime}\left(u_{2}, v_{2}\right)\right)\left(u_{1}-u_{2}, v_{1}-v_{2}\right)>0 \text {; }
$$

(iii) $\Phi^{\prime}$ is a mapping of type $\left(S_{+}\right)$; that is, if $\left(u_{n}, v_{n}\right) \rightarrow(u, v)$ in $X$ and

$$
\varlimsup_{n \rightarrow \infty}\left[\Phi^{\prime}\left(u_{n}, v_{n}\right)-\Phi^{\prime}(u, v)\right]\left(u_{n}-u, v_{n}-v\right) \leq 0,
$$

then $\left(u_{n}, v_{n}\right) \rightarrow(u, v)$ in $X$;

(iv) $\Phi^{\prime}: X \rightarrow X^{*}$ is a bounded homeomorphism.

Theorem 9. If the assumption $\left(A_{0}\right)$ is satisfied, then $\Psi \in$ $C^{1}(X, \mathbb{R})$. Moreover, $\Psi$ and $\Psi^{\prime}$ are weakly-strongly continuous; that is, $\left(u_{n}, v_{n}\right) \rightarrow(u, v)$ in $X$ implies $\Psi\left(u_{n}, v_{n}\right) \rightarrow \Psi(u, v)$ and $\Psi^{\prime}\left(u_{n}, v_{n}\right) \rightarrow \Psi^{\prime}(u, v)$ in $X^{*}$.

Proof. Suppose $\left\{\left(u_{n}, v_{n}\right)\right\}$ is a weak convergent sequence in $X$. From Proposition 6, we can conclude that $\left\{\left(u_{n}, v_{n}\right)\right\}$ is strong convergent in $L^{\gamma(\cdot)} \times L^{\mu(\cdot)}$, where $\gamma, \mu \in C(\bar{\Omega})$ and satisfies

$$
\begin{array}{r}
1 \leq \gamma(x)<\frac{r(x)-1}{r(x)} p^{*}(x), \\
1 \leq \mu(x)<\frac{r(x)-1}{r(x)} q^{*}(x), \\
\forall x \in \bar{\Omega} .
\end{array}
$$

From Proposition 2, we can see that $\Psi$ is weakly-strongly continuous, and $\Psi^{\prime}\left(u_{n}, v_{n}\right) \rightarrow \Psi^{\prime}(u, v)$ in $X^{*}$.

Since $X$ is a separable and reflexive Banach space, there are $\left\{e_{j}\right\} \subset X$ and $\left\{e_{j}^{*}\right\} \subset X^{*}$ such that

$$
\begin{gathered}
X=\overline{\operatorname{span}}\left\{e_{j}, j=1,2, \ldots\right\}, \\
X^{*}=\overline{\operatorname{span}}^{w^{*}}\left\{e_{j}^{*}, j=1,2, \ldots\right\}, \\
\left\langle e_{i}^{*}, e_{j}\right\rangle= \begin{cases}1, & i=j, \\
0, & i \neq j .\end{cases}
\end{gathered}
$$

For convenience, we write

$$
X_{j}=\operatorname{span}\left\{e_{j}\right\}, \quad Y_{k}=\bigoplus_{j=1}^{k} X_{j}, \quad Z_{k}=\overline{\bigoplus_{j=1}^{\infty} X_{j}} .
$$

Definition 10. One says that $J$ satisfies (PS) condition in $X$, if any sequence $\left\{\left(u_{n}, v_{n}\right)\right\} \subset X$ such that $\left\{J\left(u_{n}, v_{n}\right)\right\}$ is bounded and $\left\|J^{\prime}\left(u_{n}, v_{n}\right)\right\|_{*} \rightarrow 0$, as $n \rightarrow \infty$, has a convergent subsequence.

One assumes $G$ satisfies the following condition:

(B) there exist functions $\theta_{1}, \theta_{2} \in C^{1}(\bar{\Omega})$ satisfying $p(x)<$ $\theta_{1}(x)<p^{*}(x), q(x)<\theta_{2}(x)<q^{*}(x)$ on $\bar{\Omega}$ and

$$
0 \leq G(x, s, t) \leq \frac{s}{\theta_{1}} \frac{\partial}{\partial s} G(x, s, t)+\frac{t}{\theta_{2}} \frac{\partial}{\partial t} G(x, s, t),
$$

$$
\forall x \in \Omega, \quad|s|^{\theta_{1}}+|t|^{\theta_{2}} \geq 2 M .
$$

Lemma 11. If $(\lambda \geq 1)$ is large enough, $\left(A_{0}\right)$ and $(B)$ are satisfied; then $J$ satisfies (PS) condition on $X$.

Proof. It follows from (B) that

$$
\begin{array}{r}
F(x, s, t) \leq \frac{1}{\theta_{1}} s F_{s}(x, s, t)+\frac{1}{\theta_{2}} t F_{t}(x, s, t)+c_{1} a(x), \\
\forall(x, s, t) \in \bar{\Omega} \times \mathbb{R}^{2} .
\end{array}
$$

Denote

$$
\begin{aligned}
& l_{1}=\min _{x \in \bar{\Omega}}\left(\frac{1}{p(x)}-\frac{1}{\theta_{1}(x)}\right), \\
& l_{2}=\min _{x \in \bar{\Omega}}\left(\frac{1}{q(x)}-\frac{1}{\theta_{2}(x)}\right) .
\end{aligned}
$$

It is easy to see that $l_{1}, l_{2}>0$.

Let $\left\{\left(u_{n}, v_{n}\right)\right\}$ be a (PS) sequence. By computation,

$$
\begin{aligned}
c+\left(\left\|u_{n}\right\|_{p(\cdot)}+\left\|v_{n}\right\|_{q(\cdot)}\right) & \\
\geq & J\left(u_{n}, v_{n}\right)-J^{\prime}\left(u_{n}, v_{n}\right)\left(\frac{1}{\theta_{1}(x)} u_{n}, \frac{1}{\theta_{2}(x)} v_{n}\right) \\
= & \int_{\Omega} \frac{1}{p(x)}\left(\left|\nabla u_{n}\right|^{p(x)}+\lambda\left|u_{n}\right|^{p(x)}\right) d x \\
& +\int_{\Omega} \frac{1}{q(x)}\left(\left|\nabla v_{n}\right|^{q(x)}+\lambda\left|v_{n}\right|^{q(x)}\right) d x \\
& -\int_{\Omega} F\left(x, u_{n}, v_{n}\right) d x \\
& -\int_{\Omega} \frac{1}{\theta_{1}(x)}\left(\left|\nabla u_{n}\right|^{p(x)}+\lambda\left|u_{n}\right|^{p(x)}\right) d x \\
& +\int_{\Omega} \frac{1}{\theta_{2}^{2}(x)} v_{n}\left|\nabla v_{n}\right|^{q(x)-2} \nabla v_{n} \nabla \theta_{2}(x) d x \\
& +\int_{\Omega} \frac{1}{\theta_{1}(x)} u_{n} F_{u}\left(x, u_{n}, v_{n}\right) d x \\
& +\int_{\Omega} \frac{1}{\theta_{2}(x)}\left(\left|\nabla v_{n}\right|^{q(x)}+\lambda\left|v_{n}\right|^{q(x)}\right) d x \\
& +u_{1}^{2}(x) \\
& +\left.\nabla u_{n}\right|^{p(x)-2} \nabla u_{n} \nabla \theta_{1}(x) d x
\end{aligned}
$$




$$
\begin{aligned}
& \geq \int_{\Omega}\left(\frac{1}{p(x)}-\frac{1}{\theta_{1}(x)}\right)\left(\left|\nabla u_{n}\right|^{p(x)}+\lambda\left|u_{n}\right|^{p(x)}\right) d x \\
& +\int_{\Omega}\left(\frac{1}{q(x)}-\frac{1}{\theta_{2}(x)}\right)\left(\left|\nabla v_{n}\right|^{p(x)}+\lambda\left|v_{n}\right|^{p(x)}\right) d x \\
& -\int_{\Omega} \frac{\left|\nabla \theta_{1}(x)\right|}{\theta_{1}^{2}(x)}\left|u_{n}\right|\left|\nabla u_{n}\right|^{p(x)-1} d x \\
& -\int_{\Omega} \frac{\left|\nabla \theta_{2}(x)\right|}{\theta_{2}^{2}(x)}\left|v_{n}\right|\left|\nabla v_{n}\right|^{q(x)-1} d x-c_{2} \\
& \geq l_{1} \int_{\Omega}\left(\left|\nabla u_{n}\right|^{p(x)}+\lambda\left|u_{n}\right|^{p(x)}\right) d x \\
& +l_{2} \int_{\Omega}\left(\left|\nabla v_{n}\right|^{p(x)}+\lambda\left|v_{n}\right|^{p(x)}\right) d x \\
& -\int_{\Omega} \frac{\left|\nabla \theta_{1}(x)\right|}{\theta_{1}^{2}(x)}\left|u_{n}\right|\left|\nabla u_{n}\right|^{p(x)-1} d x \\
& -\int_{\Omega} \frac{\left|\nabla \theta_{2}(x)\right|}{\theta_{2}^{2}(x)}\left|v_{n}\right|\left|\nabla v_{n}\right|^{q(x)-1} d x-c_{2} .
\end{aligned}
$$

From Young inequality, we have

$$
\begin{aligned}
& \frac{\left|\nabla \theta_{1}(x)\right|}{\theta_{1}^{2}(x)}\left|u_{n}\right|\left|\nabla u_{n}\right|^{p(x)-1} \\
& \leq C_{1} \frac{1}{p(x)}\left(\frac{1}{\varepsilon_{1}}\left|u_{n}\right|\right)^{p(x)} \\
& \quad+C_{1} \frac{p(x)-1}{p(x)}\left(\varepsilon_{1}\left|\nabla u_{n}\right|^{p(x)-1}\right)^{p(x) /(p(x)-1)} \\
& =C_{1} \frac{1}{p(x)} \frac{1}{\varepsilon_{1}^{p(x)}}\left|u_{n}\right|^{p(x)} \\
& \quad+C_{1} \frac{p(x)-1}{p(x)} \varepsilon_{1}^{p(x) /(p(x)-1)}\left|\nabla u_{n}\right|^{p(x)} .
\end{aligned}
$$

Similarly, we have

$$
\begin{aligned}
& \frac{\left|\nabla \theta_{2}(x)\right|}{\theta_{2}^{2}(x)}\left|v_{n}\right|\left|\nabla v_{n}\right|^{q(x)-1} \\
& \leq C_{2} \frac{1}{q(x)} \frac{1}{\varepsilon_{1}^{q(x)}}\left|v_{n}\right|^{q(x)} \\
& \quad+C_{2} \frac{q(x)-1}{q(x)} \varepsilon_{1}^{q(x) /(q(x)-1)}\left|\nabla v_{n}\right|^{q(x)} .
\end{aligned}
$$

Assume $\varepsilon_{1}$ is a small enough positive constant and $\lambda \geq 1$ is large enough; we have

$$
\begin{aligned}
c+( & \left.\left\|u_{n}\right\|_{p(\cdot)}+\left\|v_{n}\right\|_{q(\cdot)}\right) \\
\geq & J\left(u_{n}, v_{n}\right)-J^{\prime}\left(u_{n}, v_{n}\right)\left(\frac{1}{\theta_{1}(x)} u_{n}, \frac{1}{\theta_{2}(x)} v_{n}\right) \\
\geq & \frac{2 l_{1}}{3} \int_{\Omega}\left(\left|\nabla u_{n}\right|^{p(x)}+\lambda\left|u_{n}\right|^{p(x)}\right) d x \\
& +\frac{2 l_{2}}{3} \int_{\Omega}\left(\left|\nabla v_{n}\right|^{p(x)}+\lambda\left|v_{n}\right|^{p(x)}\right) d x-C .
\end{aligned}
$$

Thus $\left\{\left\|u_{n}\right\|_{p(\cdot)}\right\}$ and $\left\{\left\|v_{n}\right\|_{q(\cdot)}\right\}$ are bounded. Therefore $\left\{\left(u_{n}, v_{n}\right)\right\}$ has a weak convergent subsequence (which we still denote by $\left.\left\{\left(u_{n}, v_{n}\right)\right\}\right)$ such that $\left(u_{n}, v_{n}\right) \rightarrow(u, v)$ as $n \rightarrow+\infty$. According to Theorem 9, we have $\Psi^{\prime}\left(u_{n}, v_{n}\right) \rightarrow \Psi^{\prime}(u, v)$ as $n \rightarrow+\infty$. Since $J^{\prime}\left(u_{n}, v_{n}\right)=\Phi^{\prime}\left(u_{n}, v_{n}\right)-\Psi^{\prime}\left(u_{n}, v_{n}\right) \rightarrow 0$ as $n \rightarrow+\infty$, we have $\Phi^{\prime}\left(u_{n}, v_{n}\right) \rightarrow \Psi^{\prime}(u, v)$ as $n \rightarrow+\infty$. Since $\Phi^{\prime}$ is a homeomorphism, we have that $\left\{\left(u_{n}, v_{n}\right)\right\}$ is strong convergent in $X$.

\section{Existence and Multiplicity of Solutions}

In this section, using the critical point theory, we will discuss the existence and multiple existence of constant sign solutions of problem $(\mathrm{P})$.

Definition 12. One calls $(u, v) \in X$ is a weak solution of $(\mathrm{P})$ if

$$
\begin{array}{r}
\int_{\Omega}|\nabla u|^{p(x)-2} \nabla u \cdot \nabla \varphi d x+\int_{\Omega} \lambda|u|^{p(x)-2} u \cdot \varphi d x \\
=\int_{\Omega} F_{u}(x, u, v) \varphi d x, \quad \forall \varphi \in W^{1, p(\cdot)}(\Omega), \\
\int_{\Omega}|\nabla v|^{q(x)-2} \nabla v \cdot \nabla \psi d x+\int_{\Omega} \lambda|v|^{q(x)-2} v \cdot \psi d x \\
=\int_{\Omega} F_{v}(x, u, v) \psi d x, \quad \forall \psi \in W^{1, q(\cdot)}(\Omega) .
\end{array}
$$
(P).

It is easy to see that the critical point of $J$ is a solution of

Denote $G^{+}(x, u, v)=G(x, S(u), S(v)), F^{+}(x, u, v)=$ $F(x, S(u), S(v))$, where $S(t)=\max \{0, t\}$. For any $(u, v) \in X$, we say $(u, v)$ belong to the first, the second, the third, or the fourth quadrant of $X$, if $u \geq 0$ and $v \geq 0, u \leq 0$ and $v \geq 0$, $u \leq 0$ and $v \leq 0$, and $u \geq 0$ and $v \leq 0$, respectively.

Definition 13. (i) One calls that $F$ satisfies sub- $(p(x), q(x))$ growth condition near the origin in the first quadrant of $X$, if it satisfies the following.

$\left(\mathrm{A}_{1}\right)$

$$
\begin{aligned}
& F(x, s, t) \geq \sigma\left(|s|^{\epsilon_{1}(x)}+|t|^{\epsilon_{2}(x)}\right), \\
& \forall(x, s, t) \in \bar{\Omega}_{0} \times(0, \delta) \times(0, \delta),
\end{aligned}
$$

where $1 \leq \epsilon_{1}(x)<p(x), 1 \leq \epsilon_{2}(x)<q(x)$ on $\bar{\Omega}_{0}$. 
(ii) One calls that $F$ satisfies super- $(p(x), q(x))$ growth condition near the infinity in the first quadrant of $X$, if it satisfies

$$
\left(\mathrm{A}_{2}\right) 0<G_{i}(x, s, t) \leq\left(1 / \theta_{1}\right) s G_{s}(x, s, t)+
$$
$\left(1 / \theta_{2}\right) t G_{t}(x, s, t)$ for $x \in \bar{\Omega}$ and $|s|^{\theta_{1}}+|t|^{\theta_{2}} \geq 2 M>0$ with $s, t>0$.

Remark. (i) Similarly, we can give the definitions of $F$ satisfying sub- $(p(x), q(x))$ growth condition near the origin, super- $(p(x), q(x))$ growth condition near the origin or near the infinity in the second, the third, and the fourth quadrant of $X$, respectively.

(ii) We say $F$ satisfies sub- $(p(x), q(x))$ growth condition near the origin, super- $(p(x), q(x))$ growth condition near the infinity in $X$, if $F$ satisfies corresponding growth condition in every quadrant of $X$.

(iii) We say $F$ satisfies some growth condition in local, if it satisfies some growth condition in a quadrant.

We will discuss the existence of solutions in the following three cases:

Case (I): $F$ satisfies sub- $(p(x), q(x))$ growth condition near the origin in local;

Case (II): $F$ satisfies super- $(p(x), q(x))$ growth condition near the infinity in local;

Case (III): $F$ satisfies sub- $(p(x), q(x))$ growth condition near the origin and super- $(p(x), q(x))$ growth condition near the infinity in local.

\subsection{Case (I). We assume}

(S) $F$ satisfies $F_{s}(x, s, t)=F_{t}(x, s, t)=0, \forall x \in \bar{\Omega}, \forall s, t \in$ $\mathbb{R}$ with $s t=0$.

Theorem 14. If $\lambda>0$ is large enough and $F$ satisfies $\left(A_{0}\right)$, $(S)$, and sub- $(p(x), q(x))$ growth condition near the origin in the first quadrant of $X$, then the problem $(P)$ has a nontrivial constant sign solution in the first quadrant of $X$.

Proof. It is easy to check that $G^{+}(x, s, t) \in C^{1}\left(\bar{\Omega} \times \mathbb{R}^{2}, \mathbb{R}\right)$, and

$$
\begin{aligned}
& F_{u}^{+}(x, u, v)=F_{u}(x, S(u), S(v)), \\
& F_{v}^{+}(x, u, v)=F_{v}(x, S(u), S(v)) .
\end{aligned}
$$

Let us consider the following auxiliary problem:

$$
\begin{aligned}
& -\operatorname{div}\left(|\nabla u|^{p(x)-2} \nabla u\right)+\lambda|u|^{p(x)-2} u=F_{u}^{+}(x, u, v) \quad \text { in } \Omega \text {, } \\
& -\operatorname{div}\left(|\nabla v|^{q(x)-2} \nabla v\right)+\lambda|v|^{q(x)-2} v=F_{v}^{+}(x, u, v) \quad \text { in } \Omega \text {, } \\
& \frac{\partial u}{\partial \gamma}=0=\frac{\partial v}{\partial \gamma} \quad \text { on } \partial \Omega
\end{aligned}
$$

The corresponding functional is

$$
J^{+}(u, v)=\Phi(u, v)-\Psi^{+}(u, v), \quad \forall(u, v) \in X
$$

where

$$
\begin{aligned}
\Psi^{+}(u, v) & =\int_{\Omega} F^{+}(x, u, v) d x \\
& =\int_{\Omega} F(x, S(u), S(v)) d x, \quad \forall(u, v) \in X .
\end{aligned}
$$

We divide $\Omega$ into several disjoint subsets $\Omega_{1}, \ldots, \Omega_{n_{0}}$ which satisfy

$$
\begin{aligned}
& \min _{x \in \overline{\Omega_{j}}} \frac{p^{*}(x)}{r^{0}(x)}>\max _{x \in \overline{\Omega_{j}}} \alpha(x)>\max _{x \in \overline{\Omega_{j}}} p(x), \quad j=1, \ldots, n_{0}, \\
& \min _{x \in \overline{\Omega_{j}}} \frac{q^{*}(x)}{r^{0}(x)}>\max _{x \in \overline{\Omega_{j}}} \beta(x)>\max _{x \in \overline{\Omega_{j}}} q(x), \quad j=1, \ldots, n_{0} .
\end{aligned}
$$

In the following, we denote

$$
\begin{array}{r}
f_{j}^{-}=\min _{x \in \overline{\Omega_{j}}} f(x), \quad f_{j}^{+}=\max _{x \in \overline{\Omega_{j}}} f(x) \\
j=1, \ldots, n_{0}, \quad \forall f \in C(\bar{\Omega}), \\
\Phi_{\Omega_{j}}(u, v)=\int_{\Omega_{j}} \frac{1}{p(x)}\left(|\nabla u|^{p(x)}+\lambda|u|^{p(x)}\right) d x \\
+\int_{\Omega_{j}} \frac{1}{q(x)}\left(|\nabla v|^{q(x)}+\lambda|v|^{q(x)}\right) d x, \\
\forall(u, v) \in X .
\end{array}
$$

Suppose $\|(u, v)\|=\delta$ is small enough. By $\left(\mathrm{A}_{0}\right)$ and Proposition 6, we have

$$
\begin{gathered}
J^{+}(u, v)=\sum_{j=1}^{n_{0}}\left\{\Phi_{\Omega_{j}}(u, v)-\int_{\Omega_{j}} F^{+}(x, u, v) d x\right\} \\
\geq \sum_{j=1}^{n_{0}}\left\{\Phi_{\Omega_{j}}(u, v)\right. \\
-\int_{\Omega_{j}} a(x)[(|u|+|v|) \\
\left.\left.+C\left(|u|^{\alpha_{j}^{+}}+|v|^{\beta_{j}^{+}}\right)\right] d x\right\} \\
\geq \sum_{j=1}^{n_{0}}\left\{\Phi_{\Omega_{j}}(u, v)-\int_{\Omega_{j}} a(x)(|u|+|v|) d x\right. \\
\left.-C\left(\|u\|^{\alpha_{j}^{+}}+\|v\|^{\beta_{j}^{+}}\right)\right\} .
\end{gathered}
$$


Note that $\alpha_{j}^{+}>p_{j}^{+}$and $\beta_{j}^{+}>q_{j}^{+}$. When $\|(u, v)\|=\delta$ is small enough, it follows from Proposition 3 that

$$
\begin{aligned}
J^{+}(u, v) \geq & \sum_{j=1}^{n_{0}}\left\{\frac{1}{2} \Phi_{\Omega_{j}}(u, v)-\int_{\Omega_{j}} a(x)(|u|+|v|) d x\right\} \\
\geq & \frac{1}{2} \Phi(u, v)-\int_{\Omega} a(x)(|u|+|v|) d x \\
\geq & \frac{1}{2 p^{+}}\|u\|_{p(\cdot)}^{p^{+}}+\frac{1}{2 q^{+}}\|v\|_{q(\cdot)}^{q^{+}}+\frac{\lambda-1}{2 p^{+}}|u|_{p(\cdot)}^{p^{+}} \\
& +\frac{\lambda-1}{2 q^{+}}|u|_{q(\cdot)}^{q^{+}}-\int_{\Omega} a(x)(|u|+|v|) d x .
\end{aligned}
$$

Obviously,

$$
\begin{aligned}
\int_{\Omega}|a|(|u|+|v|) d x & \leq\left(|a|_{p^{0}(\cdot)}|u|_{p(\cdot)}+|a|_{q^{0}(\cdot)}|v|_{q(\cdot)}\right) \\
& \leq\left(|a|_{p^{0}(\cdot)}+|a|_{q^{0}(\cdot)}\right)\left(|u|_{p(\cdot)}+|v|_{q(\cdot)}\right) \\
& =: C_{*}\left(|u|_{p(\cdot)}+|v|_{q(\cdot)}\right) .
\end{aligned}
$$

Noting that $\|(u, v)\|=\max \left\{\|u\|_{p(\cdot)},\|v\|_{q(\cdot)}\right\}=\delta$, without loss of generality, we may assume that $\|u\|_{p(\cdot)}=\delta$.

Assume $C_{*}|u|_{p} \leq\left(1 / 4 p^{+}\right)\|u\|_{p(\cdot)}^{p^{+}}$. Obviously

$$
\frac{1}{2 p^{+}}\|u\|_{p(\cdot)}^{p^{+}}-C_{*}|u|_{p} \geq \frac{1}{4 p^{+}}\|u\|_{p(\cdot)}^{p^{+}}
$$

Assume $C_{*}|u|_{p(\cdot)}>\left(1 / 4 p^{+}\right)\|u\|_{p(\cdot)}^{p^{+}}=\left(1 / 4 p^{+}\right) \delta^{p^{+}}$. When $\lambda$ is large enough, then we have

$$
\frac{\lambda-1}{2 p^{+}}|u|_{p(\cdot)}^{p^{+}} \geq \frac{\lambda-1}{2 p^{+}}\left(\frac{1}{4 p^{+} C_{*}} \delta^{p^{+}}\right)^{p^{+}} \geq 4 C_{*} \delta \geq 4 C_{*}|u|_{p(\cdot)}
$$

Thus, when $\lambda$ is large enough, we have

$$
\frac{1}{2 p^{+}}\|u\|_{p(\cdot)}^{p^{+}}+\frac{\lambda-1}{2 p^{+}}|u|_{p(\cdot)}^{p^{+}}-C_{*}|u|_{p} \geq \frac{1}{4 p^{+}}\|u\|_{p(\cdot)}^{p^{+}}=\frac{1}{4 p^{+}} \delta^{p^{+}} .
$$

Assume $C_{*}|v|_{q(\cdot)} \leq\left(1 / 8 p^{+}\right)\|u\|_{p(\cdot)}^{p^{+}}=\left(1 / 8 p^{+}\right) \delta^{p^{+}}$. We have

$$
\begin{gathered}
\frac{1}{2 p^{+}}\|u\|_{p(\cdot)}^{p^{+}}+\frac{1}{2 q^{+}}\|v\|_{q(\cdot)}^{q^{+}}+\frac{\lambda-1}{2 p^{+}}|u|_{p(\cdot)}^{p^{+}}+\frac{\lambda-1}{2 q^{+}}|u|_{q(\cdot)}^{q^{+}} \\
-C_{*}\left(|u|_{p}+|v|_{q}\right) \\
\geq \frac{1}{4 p^{+}} \delta^{p^{+}}-C_{*}|v|_{q(\cdot)} \geq \frac{1}{8 p^{+}} \delta^{p^{+}} .
\end{gathered}
$$

Assume $C_{*}|v|_{q(\cdot)} \geq\left(1 / 8 p^{+}\right)\|u\|_{p(\cdot)}^{p^{+}}=\left(1 / 8 p^{+}\right) \delta^{p^{+}}$. Similar to the above discussion, when $\lambda$ is large enough, we have

$$
\begin{aligned}
\frac{1}{2 p^{+}} & \|u\|_{p(\cdot)}^{p^{+}}+\frac{1}{2 q^{+}}\|v\|_{q(\cdot)}^{q^{+}}+\frac{\lambda-1}{2 p^{+}}|u|_{p(\cdot)}^{p^{+}}+\frac{\lambda-1}{2 q^{+}}|u|_{q(\cdot)}^{q^{+}} \\
& -C_{*}\left(|u|_{p}+|v|_{q}\right) \\
\geq & \frac{1}{4 q^{+}}\|u\|_{p(\cdot)}^{p^{+}}
\end{aligned}
$$

Therefore, when $\lambda$ is large enough, we have

$$
\begin{array}{r}
J^{+}(u, v) \geq \max \left\{\frac{1}{4 p^{+}}\|u\|_{p(\cdot)}^{p^{+}}, \frac{1}{4 q^{+}}\|v\|_{q(\cdot)}^{q^{+}}\right\}>C>0, \\
\text { when }\|(u, v)\|=\delta .
\end{array}
$$

It is easy to see that $J^{+}$is weak lower semicontinuous. Therefore, $J^{+}(u, v)$ can archive its infimum at some point $\left(u_{*}, v_{*}\right)$. Obviously, $\left(u_{*}^{-}, v_{*}^{-}\right):=\left(S\left(-u_{*}\right), S\left(-v_{*}\right)\right) \in X$. Taking $\left(u_{*}^{-}, v_{*}^{-}\right)$as a test function for $\left(\mathrm{P}^{+}\right)$, it is easy to see that $\left(u_{*}^{-}, v_{*}^{-}\right)=(0,0)$. Thus $\left(u_{*}, v_{*}\right)$ is nonnegative, and then it is a constant sign solution of $(\mathrm{P})$.

Note that (50) is satisfied. Without loss of generality, we may assume that

$$
\max _{x \in \bar{\Omega}_{0}} \epsilon_{1}(x)<\min _{x \in \bar{\Omega}_{0}} p(x), \quad \max _{x \in \bar{\Omega}_{0}} \epsilon_{2}(x)<\min _{x \in \bar{\Omega}_{0}} q(x) .
$$

Take $u_{0}, v_{0} \in C_{0}^{2}\left(\bar{\Omega}_{0}\right)$ which are nontrivial nonnegative. It is easy to see that

$$
\begin{aligned}
J^{+}\left(t u_{0}, t v_{0}\right) & =\Phi\left(t u_{0}, t v_{0}\right)-\Psi^{+}\left(t u_{0}, t v_{0}\right) \\
& \leq \Phi\left(t u_{0}, t v_{0}\right)-\sigma \int_{\Omega}\left(\left|t u_{0}\right|^{\epsilon_{1}(x)}+\left|t v_{0}\right|^{\epsilon_{2}(x)}\right) d x \\
& <0 \text { as } t \longrightarrow 0^{+} .
\end{aligned}
$$

Thus $J(u, v)$ has at least one nontrivial critical point $\left(u_{*}, v_{*}\right)$ in the first quadrant of $X$ with $J\left(u_{*}, v_{*}\right)<0$. Thus, $\left(u_{*}, v_{*}\right)$ is a nontrivial constant sign solution of $(\mathrm{P})$. According to condition (S), it is easy to see that $u_{*}$ and $v_{*}$ are all nontrivial.

Theorem 15. If $\lambda>0$ is large enough and $F$ satisfies $\left(A_{0}\right)$, $(S)$, and sub- $(p(x), q(x))$ growth condition near the origin in $X$, then problem $(P)$ has at least four nontrivial constant sign solutions.

Proof. (i) Similar to the proof of Theorem 14, we can see that (P) has a nontrivial constant sign $\left(u_{i}, v_{i}\right)$ in the $i$ th quadrant of $X$, such that $J\left(u_{i}, v_{i}\right)<0, i=1,2,3,4$. According to condition (S), $u_{i}$ and $v_{i}$ are both nontrivial. Thus (P) has at least four constant sign solutions.

\subsection{Case (II)}

Theorem 16. If $\lambda>0$ is large enough and $F$ satisfies $\left(A_{0}\right),(S)$, and the super- $(p(x), q(x))$ growth condition near the infinity 
in the first quadrant of $X$, then $(P)$ has a nontrivial constant sign solution in the first quadrant of $X$.

Proof. It is easy to check that $G^{+}(x, s, t) \in C^{1}\left(\bar{\Omega} \times \mathbb{R}^{2}, \mathbb{R}\right)$ and

$$
\begin{aligned}
& F_{u}^{+}(x, u, v)=F_{u}(x, S(u), S(v)), \\
& F_{v}^{+}(x, u, v)=F_{v}(x, S(u), S(v)) .
\end{aligned}
$$

Let us consider the auxiliary problem $\left(\mathrm{P}^{+}\right)$.

The corresponding functional is

$$
J^{+}(u, v)=\Phi(u, v)-\Psi^{+}(u, v), \quad \forall(u, v) \in X,
$$

where

$$
\begin{array}{r}
\Psi^{+}(u, v)=\int_{\Omega} F^{+}(x, u, v) d x=\int_{\Omega} F(x, S(u), S(v)) d x, \\
\forall(u, v) \in X .
\end{array}
$$

We will prove that $J^{+}$satisfies the conditions of Mountain Pass Lemma.

It is easy to see that $G^{+}$satisfies

$$
0 \leq G^{+}(x, s, t) \leq \frac{1}{\theta_{1}} s G_{s}^{+}(x, s, t)+\frac{1}{\theta_{2}} t G_{t}^{+}(x, s, t)+M_{*}
$$

for $x \in \bar{\Omega}$ and $|s|^{\theta_{1}}+|t|^{\theta_{2}} \geq 2 M>0$, where

$$
\begin{gathered}
M_{*}=\max _{x \in \bar{\Omega}} \max _{|s|^{\theta_{1}}+|t|^{\theta_{2}} \leq 2 M}\left[|G(x, s, t)|+\left|s G_{s}(x, s, t)\right|\right. \\
\left.+\left|t G_{t}(x, s, t)\right|\right] .
\end{gathered}
$$

From Lemma 11, we can see that $J^{+}(u, v)$ satisfies (PS) condition in $X$.

From (29) and Proposition 7, we have

$$
\begin{gathered}
G^{+}(x, u, v) \geq c_{1}\left[\left(|u|^{\theta_{1}}+|v|^{\theta_{2}}\right)-1\right], \\
\forall(x, u, v) \in \bar{\Omega} \times[1,+\infty) \times[1,+\infty) .
\end{gathered}
$$

In the following, we denote

$$
\begin{array}{r}
f_{j}^{-}=\min _{x \in \overline{\Omega_{j}}} f(x), \quad f_{j}^{+}=\max _{x \in \overline{\Omega_{j}}} f(x), \\
j=1, \ldots, n_{0}, \quad \forall f \in C(\bar{\Omega}), \\
\Phi_{\Omega_{j}}(u, v)=\int_{\Omega_{j}} \frac{1}{p(x)}\left(|\nabla u|^{p(x)}+\lambda|u|^{p(x)}\right) d x \\
+\int_{\Omega_{j}} \frac{1}{q(x)}\left(|\nabla v|^{q(x)}+\lambda|v|^{q(x)}\right) d x, \\
\forall(u, v) \in X .
\end{array}
$$

Without loss of generality, we may assume that

$$
\theta_{1, j}^{+}<\min _{x \in \bar{\Omega}_{j}} \frac{p^{*}(x)}{r^{0}(x)}, \quad \theta_{2, j}^{+}<\min _{x \in \overline{\Omega_{j}}} \frac{q^{*}(x)}{r^{0}(x)} .
$$

Similar to the proof of Theorem 14, when $\lambda>0$ is large enough, we can get

$$
\begin{aligned}
J^{+}(u, v) & \geq \sum_{j=1}^{n_{0}} \frac{1}{4} \Phi_{\Omega_{j}}(u, v) \geq \max \left\{\frac{1}{4 p^{+}}\|u\|_{p(\cdot)}^{p^{+}}, \frac{1}{4 q^{+}}\|v\|_{q(\cdot)}^{q^{+}}\right\} \\
& >C>0, \quad \text { when }\|(u, v)\|=\delta .
\end{aligned}
$$

For fixed $\left(u_{0}, v_{0}\right) \in X$ with $u_{0}, v_{0} \geq 1$ and $t>1$, we have

$$
\begin{aligned}
J^{+}\left(t u_{0}, t v_{0}\right)= & \Phi\left(t u_{0}, t v_{0}\right)-\int_{\Omega} F^{+}\left(x, t u_{0}, t v_{0}\right) d x \\
\leq & \Phi\left(t u_{0}, t v_{0}\right) \\
& \quad-\int_{\Omega}|a(x)|\left(t^{\theta_{1}}\left|u_{0}\right|^{\theta_{1}}+t^{\theta_{2}}\left|u_{0}\right|^{\theta_{2}}\right) d x+c \\
\leq & \sum_{j=1}^{n_{0}}\left\{\Phi_{j}\left(t u_{0}, t v_{0}\right)\right. \\
& \left.\quad-\int_{\Omega_{j}}|a(x)|\left(t^{\theta_{1}}\left|u_{0}\right|^{\theta_{1}}+t^{\theta_{2}}\left|v_{0}\right|^{\theta_{2}}\right) d x\right\} \\
& +c .
\end{aligned}
$$

Since $p_{j}^{+}<\theta_{1, j}^{-}$and $q_{j}^{+}<\theta_{2, j}^{-}$on $\bar{\Omega}_{j}, J^{+}\left(t u_{0}, t v_{0}\right) \rightarrow-\infty$ as $t \rightarrow+\infty$. Obviously, $J^{+}(0,0)=0$; then $J^{+}$satisfies the conditions of Mountain Pass Lemma (see $[42,43]$ ). So, we can conclude that $J^{+}(u, v)$ has at least one nontrivial critical point $\left(u_{*}, v_{*}\right)$ with $J\left(u_{*}, v_{*}\right)>0$. Obviously, $\left(u_{*}^{-}, v_{*}^{-}\right):=$ $\left(S\left(-u_{*}\right), S\left(-v_{*}\right)\right) \in X$. Taking $\left(u_{*}^{-}, v_{*}^{-}\right)$as a test function, it is easy to see that $\left(u_{*}^{-}, v_{*}^{-}\right)=(0,0)$. Thus $\left(u_{*}, v_{*}\right)$ is nontrivial nonnegative; then it is a constant sign solution of (P). According to condition (S), it is easy to see that $u_{*}$ and $v_{*}$ are both nontrivial and satisfy $J\left(u_{*}, v_{*}\right)>0$.

Theorem 17. If $\lambda>0$ is large enough and $F$ satisfies $\left(A_{0}\right),(S)$, and the super- $(p(x), q(x))$ growth condition near infinity in $X$, then $(P)$ has four nontrivial constant sign solutions.

Proof. As $F$ satisfies the super- $(p(x), q(x))$ growth condition in $X$, then $F$ satisfies the super- $(p(x), q(x))$ growth condition in every quadrant of $X$. By Theorem 16 , we can see that $(P)$ has a solution $\left(u_{i}, v_{i}\right)$ in the $i$ th quadrant of $X$ and satisfies $J\left(u_{i}, v_{i}\right)>0$ and $u_{i}$ and $v_{i}(i=1,2,3,4)$ are both nontrivial.

\subsection{Case (III)}

Theorem 18. If $\lambda>0$ is large enough and $F$ satisfies $\left(A_{0}\right)$, $(S)$, sub- $(p(x), q(x))$ growth condition near the origin, and super- $(p(x), q(x))$ growth condition near the infinity in the first quadrant of $X$, then $(P)$ has two nontrivial constant sign solutions in the first quadrant of $X$.

Proof. Similar to the proof of Theorem 16, we can get the existence of solution $\left(u_{1}, v_{1}\right)$ in the first quadrant in $X$, which 
satisfies $J\left(u_{1}, v_{1}\right)>0$, and $u_{1}$ and $v_{1}$ both are nontrivial. By Theorem 14, (P) has the second solution $\left(u_{1}^{*}, v_{1}^{*}\right)$ in the first quadrant in $X$, which satisfies $J\left(u_{1}^{*}, v_{1}^{*}\right)<0$, and $u_{1}^{*}$ and $v_{1}^{*}$ both are nontrivial. Therefore, $(\mathrm{P})$ has at least two constant sign solutions.

Note. Let

$$
F(x, u, v)= \begin{cases}|u|^{\alpha_{1}(x)}|v|^{\beta_{1}(x)}, & u>0, v>0 \\ |u|^{\alpha_{2}(x)}|v|^{\beta_{2}(x)}, & \text { rest, }\end{cases}
$$

where $\alpha_{1}, \alpha_{2}, \beta_{1}, \beta_{2} \in C_{+}(\bar{\Omega})$ and satisfy

$$
\begin{gathered}
p(x)<\alpha_{1}(x), \quad q(x)<\beta_{1}(x), \\
\frac{\alpha_{1}(x)}{p^{*}(x)}+\frac{\beta_{1}(x)}{q^{*}(x)}<1,
\end{gathered}
$$

$\alpha_{2}(x)-p(x)$ and $\beta_{2}(x)-q(x)$ are all changed sign functions, and

$$
\frac{\alpha_{2}(x)}{p^{*}(x)}+\frac{\beta_{2}(x)}{q^{*}(x)}<1 ;
$$

then we can see that the functional $J^{+}$satisfies (PS) condition and $(\mathrm{P})$ has a nontrivial constant sign solution, but $J$ does not satisfy the Ambrosetti-Rabinowitz condition, and it is not coercive.

Theorem 19. If $\lambda>0$ is large enough and $F$ satisfies $\left(A_{0}\right),(S)$, sub- $(p(x), q(x))$ growth condition near the origin, and super$(p(x), q(x))$ growth condition near the infinity in $X$, then $(P)$ has eight nontrivial constant sign solutions in $X$.

Proof. Similar to the proof of Theorem 18, we can see that (P) has nontrivial solutions $\left(u_{i}, v_{i}\right)$ and $\left(u_{i}^{*}, v_{i}^{*}\right)$ in the $i$ th quadrant in $X$, which satisfy $J\left(u_{i}, v_{i}\right)>0$ and $J\left(u_{i}^{*}, v_{i}^{*}\right)<0$, and $u_{i}, v_{i}, u_{i}^{*}$ and $v_{i}^{*}$ are all nontrivial. Thus $(\mathrm{P})$ has at least eight nontrivial constant sign solutions.

\section{Conflict of Interests}

The author declares that there is no conflict of interests regarding the publication of this paper.

\section{Acknowledgment}

This work is partly supported by Natural Science Foundation of Ningbo (no. 2013A610276), the Professional Development Program of Zhejiang Province Visiting Scholar (no. FX2013117), National Natural Science Foundation of China (no. 11326161), and the key projects of Science and Technology Research of the Henan Education Department (no. 14A110011).

\section{References}

[1] E. Acerbi and G. Mingione, "Regularity results for a class of functionals with non-standard growth," Archive for Rational Mechanics and Analysis, vol. 156, no. 2, pp. 121-140, 2001.
[2] C. O. Alves and J. L. P. Barreiro, "Existence and multiplicity of solutions for a $p(x)$-Laplacian equation with critical growth," Journal of Mathematical Analysis and Applications, vol. 403, no. 1, pp. 143-154, 2013.

[3] P. Amorim and S. Antontsev, "Young measure solutions for the wave equation with $p(x, t)$-Laplacian: existence and blow-up," Nonlinear Analysis. Theory, Methods \& Applications, vol. 92, pp. 153-167, 2013.

[4] S. Antontsev, M. Chipot, and S. Shmarev, "Uniqueness and comparison theorems for solutions of doubly nonlinear parabolic equations with nonstandard growth conditions," Communications on Pure and Applied Analysis, vol. 12, no. 4, pp. 1527-1546, 2013.

[5] A. Coscia and G. Mingione, "Hölder continuity of the gradient of $p(x)$-harmonic mappings," Comptes Rendus de l'Académie des Sciences. Série I. Mathématique, vol. 328, no. 4, pp. 363-368, 1999.

[6] X. Fan and D. Zhao, "On the spaces $L^{p(x)}(\Omega)$ and $W^{m, p(x)}(\Omega)$," Journal of Mathematical Analysis and Applications, vol. 263, no. 2, pp. 424-446, 2001.

[7] X. Fan, "Global $C^{1, \alpha}$ regularity for variable exponent elliptic equations in divergence form," Journal of Differential Equations, vol. 235, no. 2, pp. 397-417, 2007.

[8] X. L. Fan, "On the sub-supersolution method for $p(x)$ Laplacian equations," Journal of Mathematical Analysis and Applications, vol. 330, no. 1, pp. 665-682, 2007.

[9] X. L. Fan, Y. Z. Zhao, and D. Zhao, "Compact imbedding theorems with symmetry of Strauss-Lions type for the space $W^{1, p(x)}(\Omega)$," Journal of Mathematical Analysis and Applications, vol. 255, no. 1, pp. 333-348, 2001.

[10] X.-L. Fan and Q.-H. Zhang, "Existence of solutions for $p(x)$ Laplacian Dirichlet problem," Nonlinear Analysis. Theory, Methods \& Applications, vol. 52, no. 8, pp. 1843-1852, 2003.

[11] X. L. Fan, Q. H. Zhang, and D. Zhao, "Eigenvalues of $p(x)$ Laplacian Dirichlet problem," Journal of Mathematical Analysis and Applications, vol. 302, no. 2, pp. 306-317, 2005.

[12] X. L. Fan, "Solutions for $p(x)$-Laplacian Dirichlet problems with singular coefficients," Journal of Mathematical Analysis and Applications, vol. 312, no. 2, pp. 464-477, 2005.

[13] X. L. Fan, J. S. Shen, and D. Zhao, "Sobolev embedding theorems for spaces $W^{k, p(x)}(\Omega)$," Journal of Mathematical Analysis and Applications, vol. 262, no. 2, pp. 749-760, 2001.

[14] L. Gasiński and N. S. Papageorgiou, "A pair of positive solutions for the Dirichlet $p(z)$-Laplacian with concave and convex nonlinearities," Journal of Global Optimization, vol. 56, no. 4, pp. 1347-1360, 2013.

[15] A. El Hamidi, "Existence results to elliptic systems with nonstandard growth conditions," Journal of Mathematical Analysis and Applications, vol. 300, no. 1, pp. 30-42, 2004.

[16] P. Harjulehto, V. Latvala, and O. Toivanen, "A variant of the Geman-McClure model for image restoration," Journal of Mathematical Analysis and Applications, vol. 399, no. 2, pp. 676681, 2013.

[17] P. Harjulehto, P. Hästö, and V. Latvala, "Harnack's inequality for $p(\cdot)$-harmonic functions with unbounded exponent $p$," Journal of Mathematical Analysis and Applications, vol. 352, no. 1, pp. 345-359, 2009.

[18] O. Kováčik and J. Rákosník, "On spaces $L^{p(x)}$ and $W^{k, p(x), "}$ Czechoslovak Mathematical Journal, vol. 41, no. 4, pp. 592-618, 1991. 
[19] W. L. Liu and P. H. Zhao, "Existence of positive solutions for $p(x)$-Laplacian equations in unbounded domains," Nonlinear Analysis. Theory, Methods \& Applications, vol. 69, no. 10, pp. 3358-3371, 2008.

[20] T. Lukkari, "Singular solutions of elliptic equations with nonstandard growth," Mathematische Nachrichten, vol. 282, no. 12, pp. 1770-1787, 2009.

[21] M. Mihăilescu, G. Moroşanu, and D. Stancu-Dumitru, "Equations involving a variable exponent Grushin-type operator," Nonlinearity, vol. 24, no. 10, pp. 2663-2680, 2011.

[22] M. Mihăilescu, V. Rădulescu, and D. Stancu-Dumitru, "A Caffarelli-Kohn-Nirenberg-type inequality with variable exponent and applications to PDEs," Complex Variables and Elliptic Equations, vol. 56, no. 7-9, pp. 659-669, 2011.

[23] M. Růžička, Electrorheological Fluids: Modeling and Mathematical Theory, vol. 1748 of Lecture Notes in Mathematics, Springer, 2000.

[24] S. G. Samko, "Density of $C_{0}^{\infty}\left(\mathbb{R}^{n}\right)$ in the generalized Sobolev spaces $W^{m, p(x)}\left(\mathbb{R}^{n}\right)$," Rossiǔskaya Akademiya Nauk. Doklady Akademii Nauk, vol. 369, no. 4, pp. 451-454, 1999.

[25] X. Xu and Y. An, "Existence and multiplicity of solutions for elliptic systems with nonstandard growth condition in $\mathbb{R}^{N}$," Nonlinear Analysis. Theory, Methods \& Applications, vol. 68, no. 4, pp. 956-968, 2008.

[26] Q. H. Zhang, Y. Wang, and Z. M. Qiu, "Existence of solutions and boundary asymptotic behavior of $p(r)$-Laplacian equation multi-point boundary value problems," Nonlinear Analysis. Theory, Methods \& Applications, vol. 72, no. 6, pp. 2950-2973, 2010.

[27] Q. H. Zhang, Y. R. Guo, and G. X. Chen, "Existence and multiple solutions for a variable exponent system," Nonlinear Analysis. Theory, Methods \& Applications, vol. 73, no. 12, pp. 3788-3804, 2010.

[28] Q. H. Zhang, "Existence of positive solutions to a class of $p(x)$-Laplacian equations with singular nonlinearities," Applied Mathematics Letters, vol. 25, no. 12, pp. 2381-2384, 2012.

[29] Q. H. Zhang, Z. M. Qiu, and R. Dong, "Existence and asymptotic behavior of positive solutions for a variable exponent elliptic system without variational structure," Nonlinear Analysis. Theory, Methods \& Applications, vol. 72, no. 1, pp. 354-363, 2010.

[30] Q. H. Zhang, "Existence, nonexistence and asymptotic behavior of boundary blow-up solutions to $p(x)$-Laplacian problems with singular coefficient," Nonlinear Analysis. Theory, Methods \& Applications, vol. 74, no. 6, pp. 2045-2061, 2011.

[31] V. V. Zhikov, "Averaging of functionals of the calculus of variations and elasticity theory," Izvestiya Akademii Nauk SSSR. Seriya Matematicheskaya, vol. 29, pp. 33-36, 1987.

[32] V. V. Zhikov, "Existence theorem for some pairs of coupled elliptic equations," Doklady Mathematics, vol. 77, no. 1, pp. 8084, 2008.

[33] Y. Chen, S. Levine, and M. Rao, "Variable exponent, linear growth functionals in image restoration," SIAM Journal on Applied Mathematics, vol. 66, no. 4, pp. 1383-1406, 2006.

[34] D. D. Hai and R. Shivaji, "An existence result on positive solutions for a class of $p$-Laplacian systems," Nonlinear Analysis. Theory, Methods \& Applications, vol. 56, no. 7, pp. 1007-1010, 2004.

[35] M. Chhetri and P. Girg, "Existence of positive solutions for a class of superlinear semipositone systems," Journal of Mathematical Analysis and Applications, vol. 408, no. 2, pp. 781-788, 2013.
[36] T.-S. Hsu, "Multiple positive solutions for quasilinear elliptic problems involving concave-convex nonlinearities and multiple Hardy-type terms," Acta Mathematica Scientia B. English Edition, vol. 33, no. 5, pp. 1314-1328, 2013.

[37] L. B. Jin and C. M. Li, "Positive solutions to a class of secondorder semilinear elliptic equations in an exterior domain," Nonlinear Analysis. Theory, Methods \& Applications, vol. 79, pp. 233-237, 2013.

[38] D. Motreanu, V. V. Motreanu, and N. S. Papageorgiou, "A degree theoretic approach for multiple solutions of constant sign for nonlinear elliptic equations," Manuscripta Mathematica, vol. 124, no. 4, pp. 507-531, 2007.

[39] N. S. Papageorgiou and F. Papalini, "Constant sign and nodal solutions for logistic-type equations with equidiffusive reaction," Monatshefte für Mathematik, vol. 165, no. 1, pp. 91-116, 2012.

[40] S. Carl and D. Motreanu, "Constant-sign and sign-changing solutions for nonlinear eigenvalue problems," Nonlinear Analysis. Theory, Methods \& Applications, vol. 68, no. 9, pp. 26682676, 2008.

[41] K. C. Chang, Critical Point Theory and Applications, Shanghai Scientific and Technology Press, Shanghai, China, 1986.

[42] M. Struwe, Variational Methods, Springer, Berlin, Germany, 1990.

[43] M. Willem, Minimax Theorems, Birkhäuser, Boston, Mass, USA, 1996. 


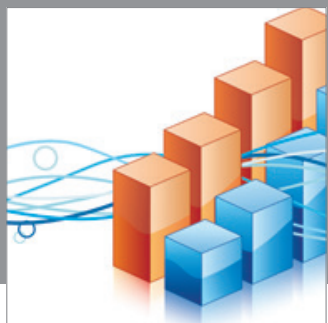

Advances in

Operations Research

mansans

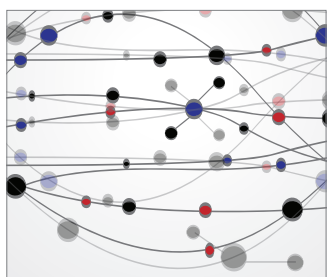

The Scientific World Journal
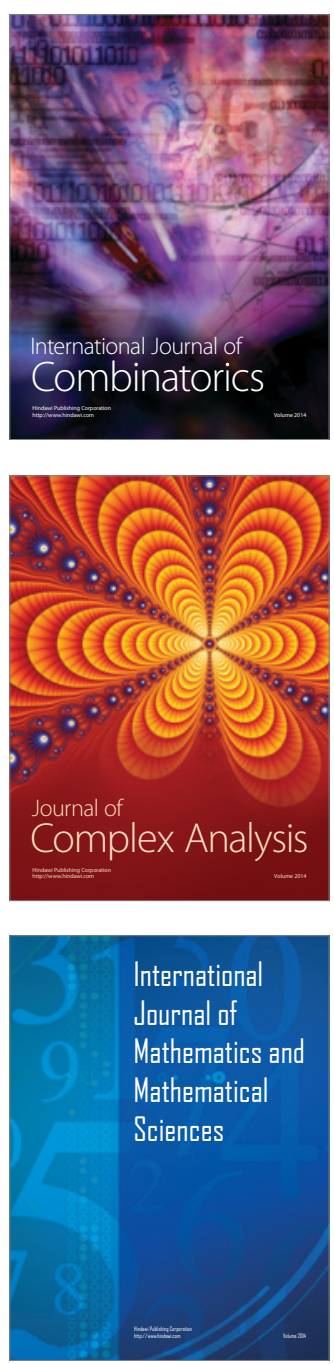
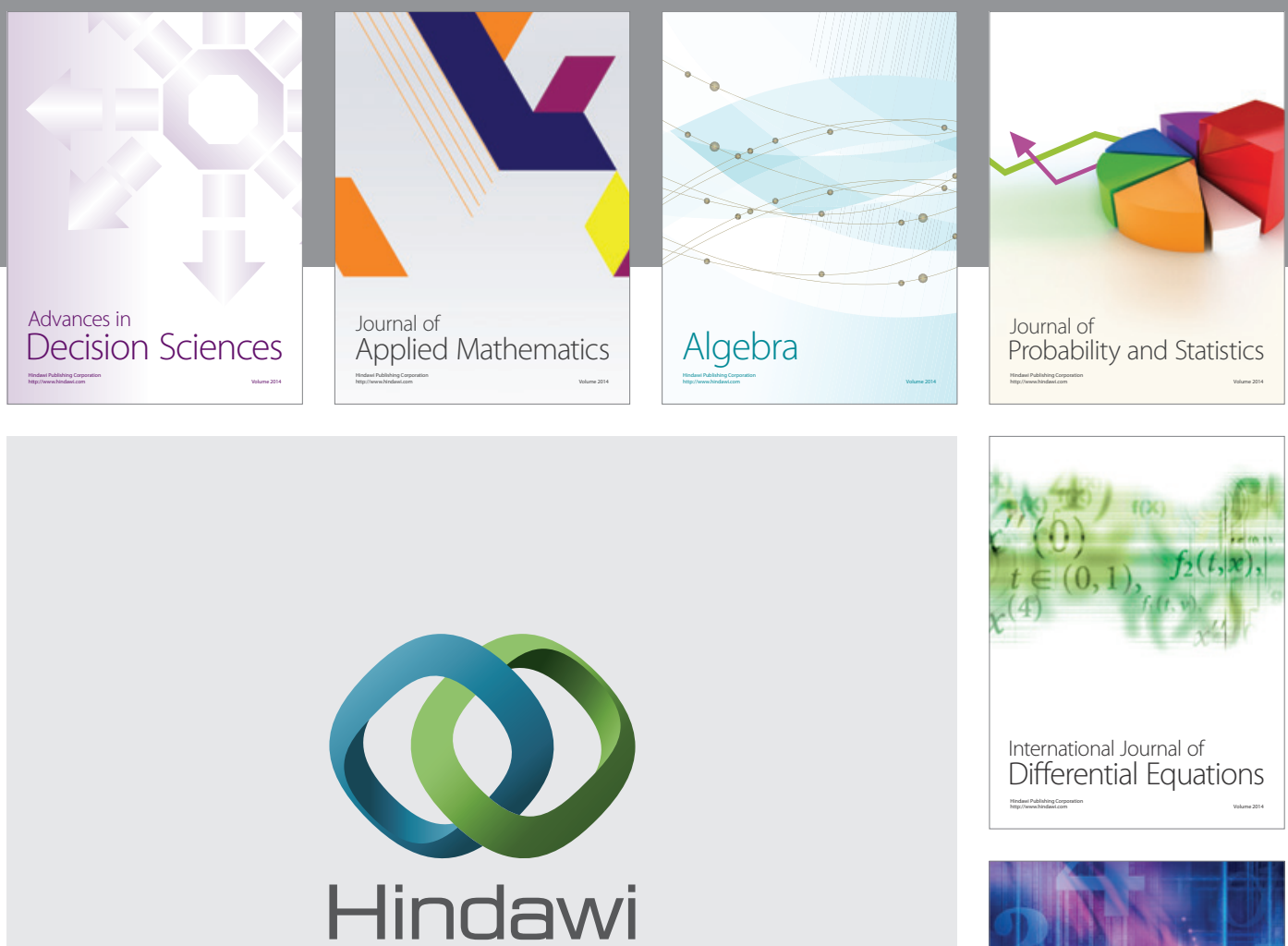

Submit your manuscripts at http://www.hindawi.com
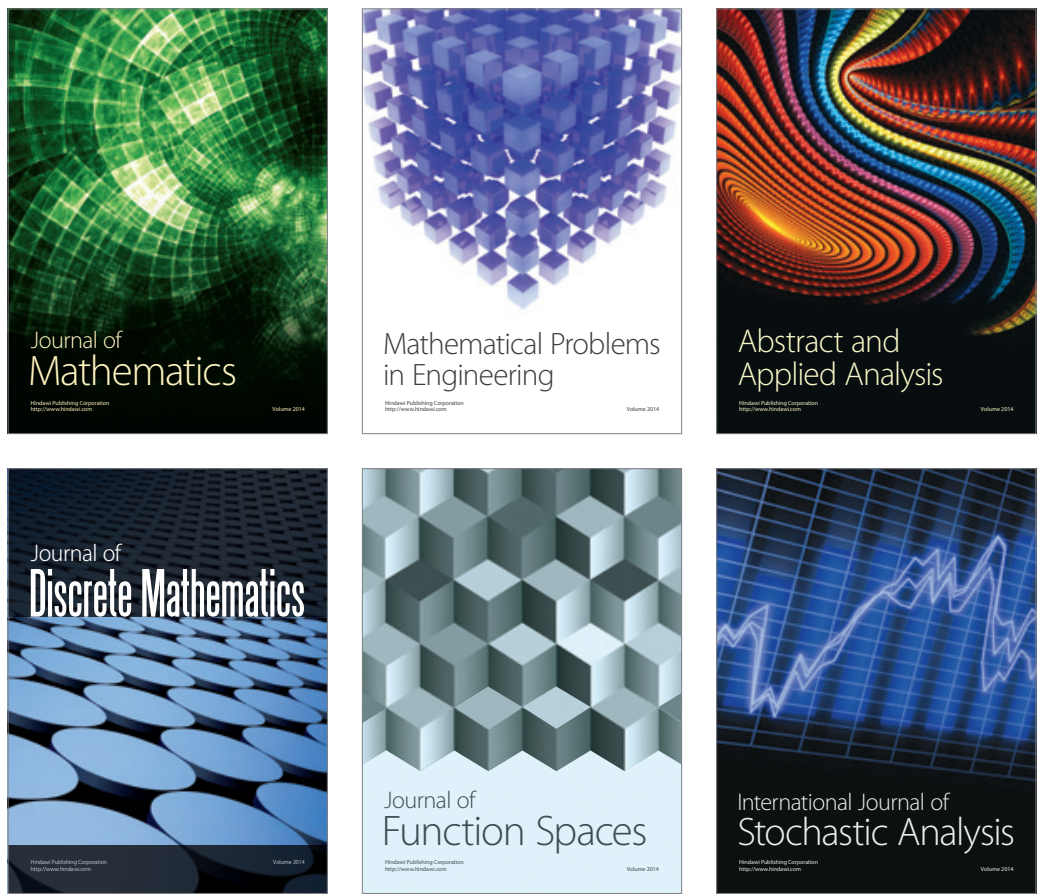

Journal of

Function Spaces

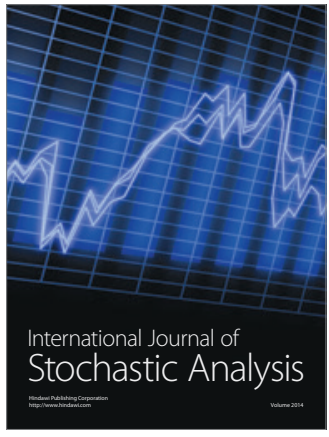

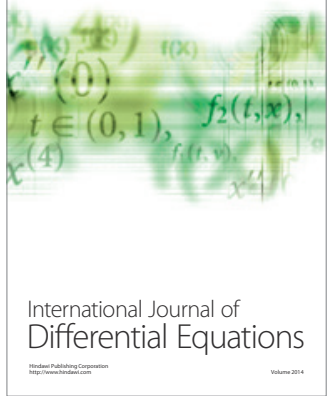
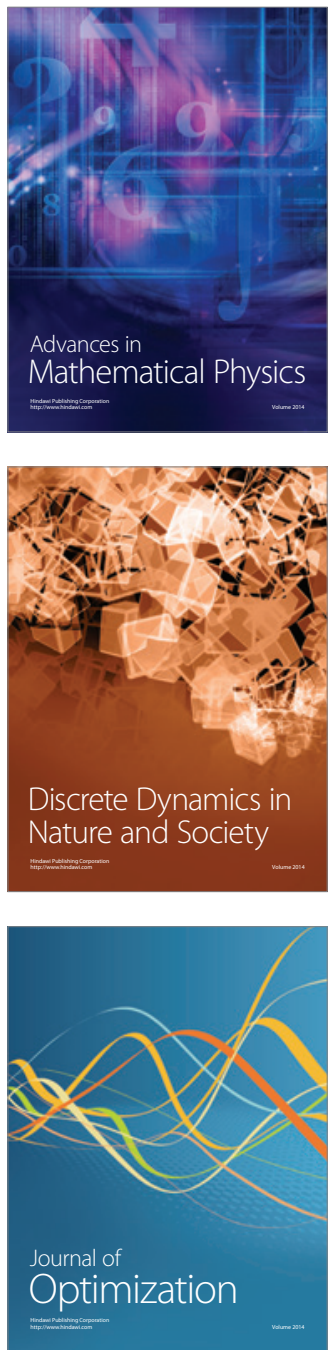